\title{
Pharmacokinetic and Drug Absorption Profiles of the Anti-Hyperglycaemic Agent Gliclazide in Oral Tissue-Targeted Microcapsules in Rats
}

\author{
Jelena Jovic, ${ }^{1}$ Boris Milijasevic, ${ }^{1}$ Sasa Vukmirovic, ${ }^{1}$ Velibor Vasovic, ${ }^{1}$ Momir Mikov, ${ }^{1}$ \\ Armin Mooranian, ${ }^{2}$ Hani Al-Salami, ${ }^{2}$ Svetlana Golocorbin-Kon ${ }^{3}$
}

\section{Abstract}

Background/Aim: Gliclazide is one of the most commonly prescribed oral anti-hyperglycaemic therapies in type 2 diabetes mellitus (T2D). Recently reported additional beneficial pharmacological properties of gliclazide, including immunomodulatory and anticoagulant activities, suggested its potential application in treatment of type 1 diabetes mellitus (T1D). However, following oral administration, gliclazide was shown to have poor and variable absorption directing research into development of novel pharmaceutical delivery systems of gliclazide suitable for T1D. Since bile acids have previously demonstrated stabilising and controlled-release effects on microcapsules, their use for preparation of microcapsules of gliclazide may lead to improvements in gliclazide release, absorption and antidiabetic effects. This investigation was aimed to evaluate drug absorption profiles and hypoglycaemic effects of alginate-based microcapsules of gliclazide, prepared together with or without cholic acid, in healthy rats.

Methods: Thirty healthy Wistar rats with confirmed normal glucose blood concentration were allocated into five groups and administered with a single dose of either vehicle microcapsules, gliclazide in suspension, gliclazide microcapsules, gliclazide in suspension together with cholic acid or microencapsulated gliclazide in combination with cholic acid. Following respective gliclazide dose, blood was sampled over next 10 hours and blood glucose levels and gliclazide serum concentrations were measured.

Results: This analysis demonstrated altered effects of different gliclazide formulations in healthy rats with the highest gliclazide absorption mirrored by the most profound hypoglycaemic effect being achieved after its oral administration as a suspension ( $p<0.01)$ compared to any other investigated pharmaceutical formulation.

Concusion:When conducting pharmacokinetic characterisation of novel pharmaceutical formulations of antidiabetic drugs, it is of utmost importance to select the appropriate research model and consider the possible role of gut-metabolic activation on their hypoglycaemic effects.

Key words: gliclazide; capsules; bile acids; hypoglycaemic agents; rats.
(1) Department of Pharmacology and Toxicology, Faculty of Medicine, University of Novi Sad, Novi Sad, Serbia

(2) Biotechnology and Drug Development Research Laboratory, School of Pharmacy and Biomedical Sciences, Curtin Health Innovation Research Institute, Curtin University, Perth, Australia

(3) Department of Pharmacy, Faculty of Medicine, University of Novi Sad, Novi Sad, Serbia.
Correspondence:

JELENA JOVIĆ

E: joviceva.jelena@gmail.com

M: +381638936683

\section{ARTICLE INFO}

Received: 2 March 2020

Revision received: 16 March 2020 Accepted: 17 March 2020

\section{Introduction}

Gliclazide, a second-generation sulphonylurea, is the second most commonly prescribed oral antihyperglycemic agent in the treatment of type
2 diabetes mellitus (T2D), after metformin. ${ }^{1}$ It is the preferred therapeutic option because of its selective binding to the pancreatic $\beta$-cell sul- 
phonylurea receptor (SUR1) and subsequent stimulation of insulin release, as well as for its unique antioxidant properties ${ }^{2}$ and other beneficial haematological effects. ${ }^{3,4}$ Furthermore, gliclazide was shown to restore peripheral insulin sensitivity, decrease hepatic glucose production and skeletal muscle glycogenesis, independent of its insulin-mediated effects. ${ }^{5}$ Due to its known extrapancreatic action, gliclazide may exert conceivable potential in type 1 diabetes mellitus (T1D) treatment, especially when used in combination with other agents that exhibit hypoglycaemic effects, including certain bile acids and probiotics, as previously reported. ${ }^{6-8}$

Despite the clinical experience with gliclazide being extensive and its pharmacological and pharmaceutical applications well-documented, poor and variable absorption after oral administration has limited its potential applications in T1D due to the inconsistent dose-response effects, necessitating research into novel pharmaceutical delivery systems of gliclazide suitable for T1D. 9 Therefore, besides available immediate and modified release formulations of gliclazide, ${ }^{10}$ novel pharmaceutical approaches that utilise high-scale techniques, such as drug microencapsulation are needed for enhancement of its oral absorption and potentially maintain tight blood glucose levels in diabetic patients. ${ }^{11}$

Multiple recent studies reported on the development and characterisation of novel alginate-based microencapsulated formulations of gliclazide mixed with different bile acids. Notably, these new gliclazide formulations exhibited stable colon-targeted delivery and its enhanced pharmacokinetic and pharmacodynamics effects in T1D rats when cholic, deoxycholic or taurocholic acids were added to the microencapsulated gliclazide formulation..$^{12-17}$

However, given the novelty of the designed microencapsulated gliclazide formulations, basic in vivo pharmacokinetic and pharmacodynamic studies should be also conducted in a sufficient number of clinically healthy animals, in order to validate the in vitro and in silico approaches needed to gain a more comprehensive understanding of its efficacy and safety profiles in diabetic models and ultimately patients.

Hence, this study was aimed to investigate the hypoglycaemic effects of alginate-based microcapsules of gliclazide, prepared together with or without cholic acid, in healthy rats. Focusing on the effects of microencapsulation and addition of cholic acid on gliclazide systemic absorption in healthy animals, this study complements the ongoing work aimed at investigating potential bile acids use and microencapsulation technology in the delivery and absorption of antidiabetic agents.

\section{Methods}

\section{Reagents and Materials}

Gliclazide (99.9\%), Na-alginate (98\%), and cholic acid (95\%) were supplied by Sigma Chemical Co, USA. Anhydrous calcium chloride was purchased from Scharlab S.L, Australia. The suspending gel for ultrasound-aided manipulation was obtained from Australian Medical Association, Perth, WA, Australia. Test tubes for oral gavage were purchased from Instech Laboratories, Inc., USA. Acetonitrile and HPLC-grade water were delivered by Fisher Chemical, Australia. All other chemical reagents and solvents were supplied by Merck, Australia.

\section{Microcapsules Preparation}

Fresh stock of gliclazide (40 mg/mL) and cholic acid $(4 \mathrm{mg} / \mathrm{mL})$ were prepared by dissolving the respective powders in $10 \%$ ultrasonic suspending gel, while the $2 \% \mathrm{CaCl}_{2}$ stock solution was prepared by adding $\mathrm{CaCl}_{2}$ powder to HPLC-grade water. Each stock preparation was stored at $4^{\circ} \mathrm{C}$ and used within 12 hours. Sodium alginate-based microcapsules of gliclazide and cholic acid were prepared as previously described, according to the established protocols. ${ }^{15,17}$

\section{Animal Procedures and Blood Glucose Mea- surements}

The conducted experiments were approved by the local Ethics Committee for Experimental Animals Welfare Protection of the University of Novi Sad, Serbia (approval number: 01-31/4-1/2014-01). All experiments were performed in alignment with ethical principles and guidelines for welfare of the laboratory animals. Male Wistar rats, 8-10 weeks old, were obtained from the Animal Farming Facility of the Military Medical Academy, Belgrade, Serbia and were allowed a 7-day acclimati- 
sation period and given access to food and water ad libitum. Following allocation into 5 separate groups with 5 rats per cage, animals were treated via oral gavage with a single dose of either vehicle microcapsules (control group), gliclazide suspension (G), microencapsulated gliclazide (G (MC)), gliclazide plus cholic acid suspension $(\mathrm{G}+\mathrm{CA})$ or gliclazide plus cholic acid microcapsules $(\mathrm{G}+\mathrm{CA}$ (MC)), with identical doses of gliclazide and cholic acid of $40 \mathrm{mg} / \mathrm{kg}$ and $4 \mathrm{mg} / \mathrm{kg}$, respectively across all treatment groups. After a single dose administration of the respective treatment, blood was drawn from tail vein at following time-points: $0,5,10,20,40,60,90,120,180,240,360,480$ and 600 min post-dose, for blood glucose and gliclazide concentrations measurements. Blood glucose levels were directly measured in collected samples, using ACCU-CHEK Glucose Advantage Meter (Roche).

\section{Gliclazide Serum Concentrations Measure- ments}

Gliclazide concentrations in serum were measured using high pressure liquid chromatography (HPLC) techniques based on the previously established methods. ${ }^{6}$ Shortly, $40 \mu \mathrm{L}$ of serum samples were extracted with acetonitrile in a 2:1 ratio, vortexed for 30 seconds and centrifuged $(10,000 \mathrm{rpm} / \mathrm{min})$ for 5 minutes. The supernatant was injected into the HPLC system, together with respective standards and quality control samples and analysed in the same manner. The used HPLC system was HPLC-DAD (Dionex, USA) with ODS Hypersil analytical column (100 $\mathrm{mm} \times 2.1 \mathrm{~mm}, 5 \mu \mathrm{m} ; 120 \AA$ ) from Agilent with the appropriate ODS precolumn (Agilent; $20 \mathrm{~mm} \mathrm{x}$ $2.1 \mathrm{~mm}, 5 \mu \mathrm{m}$ ). The mobile phase was $49 \%$ acetonitrile and $51 \%$ water, $\mathrm{v} / \mathrm{v}(\mathrm{pH} 2.7)$. The flow rate was $0.4 \mathrm{~mL} / \mathrm{min}$, and the analysis was performed using the wavelength of $229 \mathrm{~nm}$. Limit of detection (LOD) was $0.4 \mu \mathrm{g} / \mathrm{mL}$, limit of quantification (LOQ) was $0.5 \mu \mathrm{g} / \mathrm{mL}$, whereas the recovery of $85.83 \pm 4.02 \%$ and linearity of $0.8-500 \mu \mathrm{g} / \mathrm{mL}$ was noted.

\section{Statistical Analysis}

The D'Agostino-Pearson omnibus test was used to test for the normality of data $(\mathrm{p}=0.05)$. Linear regression and one-way ANOVA analysis followed by a post hoc Tukey's multiple comparison test was performed. Data are represented as mean \pm standard deviation (SD). GraphPad Prism version 5.0 (GraphPad Software, Inc., USA) was used and $p$ values reported where significant $(\mathrm{p}<$ 0.05 ) or highly significant $(\mathrm{p}<0.01)$.

\section{Results}

\section{Blood Glucose Measurements}

According to the D'Agostino-Pearson omnibus test, data passed the normality test ( $p>0.05)$, indicating normal distribution of data. All administered gliclazide formulations, with or without added cholic acid resulted in significantly lower blood glucose concentrations in healthy rats compared with control vehicle microcapsules, therefore indicating a strong and significant hypoglycaemic effects of orally administered gliclazide (Figure 1). However, the reduction in blood glucose levels was more profound $(\mathrm{p}<0.01)$ compared with control group when rats were treated with gliclazide suspension than with gliclazide microcapsules ( $\mathrm{p}<0.05$ ). On the other hand, when cholic acid was added to gliclazide, microencapsulated combination of gliclazide and cholic acid exerted stronger hypoglycaemic effects compared to the combination administered in suspension.

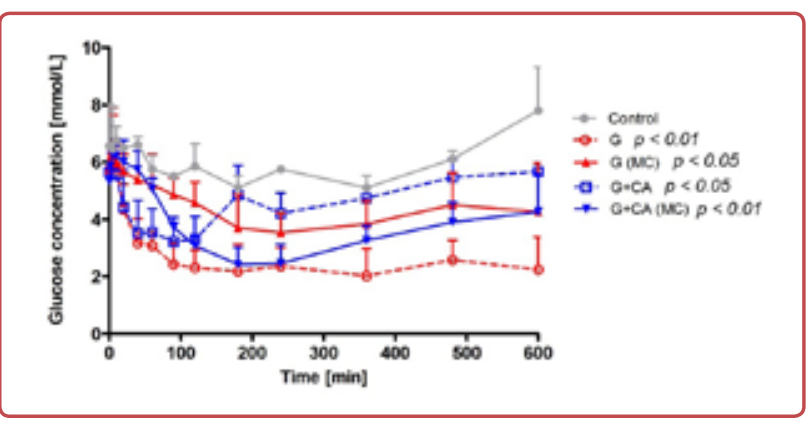

Figure 1. Blood glucose concentrations over 10 hours in five different groups of healthy rats, treated with a single dose of: empty microcapsules (untreated control), gliclazide suspension (G), gliclazide microcapsules (G (MC)), gliclazide + cholic acid suspension $(G+C A)$, gliclazide + cholic acid microcapsules $(G+C A$ $(M C))$. Data are shown as mean $\pm S D, n=5$. $p$-value vs control at all time points; one-way ANOVA followed by a post hoc Tukey's multiple comparison test.

\section{Gliclazide Serum Concentrations}

According to the D'Agostino-Pearson omnibus test, data passed the normality test ( $p>0.05)$, indicating normal distribution of data. When comparing the effect of microencapsulation technology on gliclazide serum concentrations, it is observed that microencapsulation of gliclazide resulted in its lower concentrations in serum with maximum serum concentration $\left(\mathrm{C}_{\max }\right)$ of $135.00 \pm 86.73 \mu \mathrm{g} / \mathrm{mL}$, compared with non-microencapsulated gliclazide with peak concentration in serum of $358.50 \pm 92.88 \mu \mathrm{g} / \mathrm{mL}$. (Figure 2). Moreover, the time needed for gliclazide to 
reach its peak concentration in serum $\left(\mathrm{T}_{\max }\right)$ was significantly prolonged $(\mathrm{p}<0.05)$ with microencapsulated gliclazide formulation versus gliclazide suspension, with $\mathrm{T}_{\max }$ values being $276.00 \pm 116.96$ minutes and $138.00 \pm 58.48$ min, respectively. Addition of cholic acid to gliclazide microcapsules resulted in significantly higher concentrations in serum compared to non-microencapsulated combination of gliclazide and respective bile acid $(\mathrm{p}<0.05)$.

\section{Discussion}

It has been previously shown that diabetes may influence the pharmacokinetic, as well as pharmacodynamic properties of various oral antidiabetic compounds, including gliclazide, presumably due to the pre-systemic drug elimination or altered gastric emptying and gastrointestinal motility in diabetic individuals. ${ }^{18,}{ }^{19}$ In the present study, healthy rats exhibited increased gliclazide bioavailability compared to that previously reported in diabetic rats treated with same pharmaceutical gliclazide formulations, ${ }^{17}$ regardless of its administered formulation. However, Stětinová et $\mathrm{al}^{20}$ reported that even when biological gliclazide bioavailability was similar in both healthy and alloxan-induced diabetic animals, the hypoglycaemic effect of gliclazide was not equally distributed and was less pronounced in animals with alloxan-induced hyperglycaemia (23\% decrease at $60 \mathrm{~min}$ ) compared to healthy animals (36\% decrease at $60 \mathrm{~min}$ ). Interestingly, at later time points, this gliclazide hypoglycaemic effect was maintained in hyperglycaemic animals, while healthy animals demonstrated a reversal of the hypoglycaemic effect..$^{20}$

It was shown here that in healthy normoglycaemic animals, gliclazide microencapsulation resulted in its significantly lower $\mathrm{C}_{\max }$ and almost doubled $\mathrm{T}_{\max }$ values, compared with non-microencapsulated gliclazide. On the other hand, the addition of cholic acid into the alginate-based gliclazide microcapsules increased its serum concentrations and extended the time needed to reach its peak concentration in serum compared to the combination of gliclazide plus cholic acid that was not microencapsulated (Figure 2). Similar findings have previously been reported for alloxan-induced diabetic animals. ${ }^{17}$ This was also

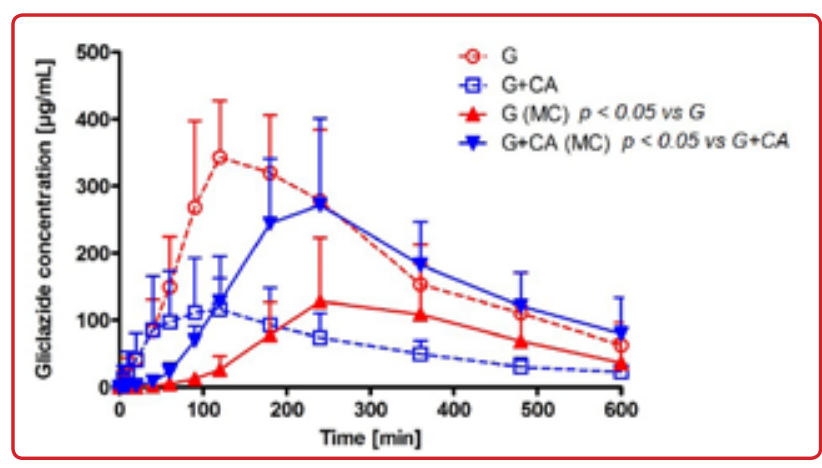

Figure 2. Gliclazide serum concentrations over time in healthy rats gavaged with a single dose of: gliclazide suspension (G), gliclazide microcapsules (G (MC)), gliclazide + cholic acid suspension $(G+C A)$, gliclazide + cholic acid microcapsules $(G+C A$ $(M C))$. Data are shown as mean $\pm S D, n=5$. $p$-value vs control at all time points; one-way ANOVA followed by a post hoc Tukey's multiple comparison test.

confirmed by the in vitro stability and release kinetic studies of the same gliclazide formulations, indicating possible suitability of microencapsulated gliclazide formulations for its sustained and targeted delivery to the lower intestine. ${ }^{12,15}$

Having a closer look at the maximum blood glucose level drops $\left(\mathrm{E}_{\max }\right)$ compared to its initial values before gliclazide administration $\left(\mathrm{E}_{\mathrm{o}}\right)$ in healthy rats, it was observed that gliclazide suspension lowered the glucose levels by $69 \%$, microencapsulated gliclazide formulation by $51 \%$, combination of gliclazide suspension and cholic acid by $46 \%$ and microencapsulated combination of gliclazide and cholic acid by $46 \%$. Interestingly, in the previously reported study in diabetic rats, ${ }^{17}$ the greatest glucose levels reduction from the initial values was observed after the administration of the microencapsulated gliclazide and not with gliclazide suspension as it was the case in healthy rats. Moreover, even though statistical test showed non-significant difference between healthy and diabetic rats, a tendency towards higher values of $\mathrm{C}_{\max }$ and shorter $\mathrm{T}_{\max }$ for gliclazide were observed in healthy rats within this study compared with alloxan-induced diabetic rats from the previous study, ${ }^{17}$ regardless of the administered gliclazide pharmaceutical formulation.

Linear regression analysis of blood glucose levels throughout time and gliclazide concentrations in serum administered in different pharmaceutical formulations in healthy rats revealed disproportional association of gliclazide suspension treatment and resulting glucose levels in blood. Even 


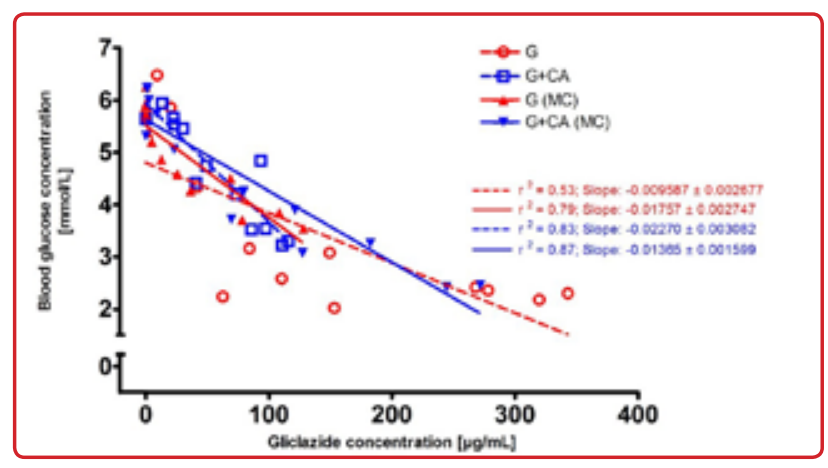

Figure 3. Linear regression of blood glucose and gliclazide serum concentrations among treatment groups of healthy rats treated with a single dose of: gliclazide suspension (G), gliclazide microcapsules (G $(M C))$, gliclazide+cholic acid suspension $(G+C A)$, gliclazide+cholic acid microcapsules $(G+C A(M C))$.

though gliclazide and cholic acid microcapsules also resulted in consistent reduction in blood glucose levels, these changes were not significantly different compared with control (Figure 3).

Mikov et $\mathrm{al}^{7}$ have reported decreased bioavailbility of gliclazide administered in suspension in alloxan-induced diabetic rats compared to healthy controls, possibly due to gastrointestinal motility disorder related to diabetes. However, in the same study, when gliclazide was administered together with sodium 3a,7a-dihydroxy12-keto- $5 \beta$-cholanate (MKC), its bioavailability was further increased in healthy animals, but no significant differences were observed in terms of glucose levels. On the other hand, gliclazide bioavailability being substantially lower in diabetic rats was not altered by this bile acid salt, but the hypoglycaemic action in diabetic rats of the combination of gliclazide and MKC was significantly greater in comparison to the situation when gliclazide was administered alone.

Differential drug permeation-enhancing and hypoglycaemic effects of various bile acids are further supported by a recently reported study that evaluated the glucose lowering potential of novel microencapsulated gliclazide pharmaceutical formulation with gliclazide alone or in combination with a taurocholic acid (TCA). Mathavan et $\mathrm{al}^{14}$ reported that microencapsulated gliclazide alone failed to exert the hypoglycaemic effect in diabetic rats, whereas the addition of TCA into gliclazide microcapsules resulted in enhanced gliclazide absorption and significant hypoglycaemic effects compared with diabetic untreated controls. However, the data on the effects of this microencapsulated gliclazide formulation in healthy animals is not reported. ${ }^{14}$
Another study that investigated pharmacokinetics and pharmacodynamics of gliclazide in immediate and modified release formulation tablets showed non-significant difference of evaluated parameters between healthy and streptozotocin-induced diabetic rats for either formulations. ${ }^{21}$ Even though the differences were not significant, a tendency towards slower elimination and higher mean residence time (MRT) in both healthy and diabetic rats treated with modified release gliclazide versus its immediate release formulation was found, confirming the sustained release mechanism of the modified release drug formulations. Resztak et ${ }^{21}$ al also reported significantly higher reduction of blood glucose level with immediate-release gliclazide formulation than with one with modified release, with the highest pharmacodynamic efficacy of gliclazide being observed in the healthy animals following treatment with the immediate-release gliclazide tablets, whereas the hypoglycaemic effect of the drug was reduced in diabetic animals. Therefore, the authors suggested that hypoglycaemic effects of gliclazide in healthy subjects might not be a suitable approach for characterising antidiabetic drugs. ${ }^{21}$

\section{Conclusion}

This study demonstrated the impact of gliclazide pharmaceutical formulations on the systemic absorption and hypoglycaemic effects in healthy rats, revealing that altered pharmacokinetic/pharmacodynamic effects were due to the modified physiological outcomes in alloxan-induced diabetic rats. The highest gliclazide absorption was achieved after its administration as a suspension and was mirrored by the most profound hypoglycaemic effect versus any other investigated pharmaceutical formulation, unlike in diabetic rats where gliclazide hypoglycaemic effects were found to be independent of its absorption and serum concentrations. Taken together, these data indicate the importance of selecting the appropriate model for pharmacokinetic characterisation of novel pharmaceutical formulations of antidiabetic drugs and the possible role of gut-metabolic activation on their hypoglycaemic effects. 


\section{Acknowledgments}

This study was funded by HORIZON 2020 MEDLEM project [Grant 690876]; Project for Scientific and Technological Development of Vojvodina [Grant 114-451-2072-/2016-02]; and Project of Ministry of Education, Science and Technological Development of the Republic of Serbia [Grant 41012].

These data were previously presented as a part of a PhD thesis of Jelena Calasan; Title: The effect of alginate microcapsules pharmaceutical formulation on gliclazide absorption in rat gastrointestinal tract.

\section{Conflict of interest}

None.

\section{References}

1. Genuth S. Should sulfonylureas remain an acceptable first-line add-on to metformin therapy in patients with type 2 diabetes? No, it's time to move on! Diabetes Care 2015;38(1):170-5.

2. Jennings PE. Vascular benefits of gliclazide beyond glycemic control. Metab Clin Exp 2000;49(10 Suppl 2):17-20.

3. Konya H, Hasegawa Y, Hamaguchi T, Satani K, Umehara A, Katsuno T, et al. Effects of gliclazide on platelet aggregation and the plasminogen activator inhibitor type 1 level in patients with type 2 diabetes mellitus. Metabolism 2010;59(9):1294-9.

4. Desfaits AC, Serri O, Renier G. Gliclazide decreases cell-mediated low-density lipoprotein (LDL) oxidation and reduces monocyte adhesion to endothelial cells induced by oxidatively modified LDL. Metab Clin Exp 1997;46(10):1150-6.

5. Sarkar A, Tiwari A, Bhasin PS, Mitra M. Pharmacological and pharmaceutical profile of gliclazide: a review. J Appl Pharm Sci 2011;1(9):11-9.

6. Al-Salami H, Butt G, Tucker I, Golocorbin-Kon S, Mikov M. Probiotics decreased the bioavailability of the bile acid analog, monoketocholic acid, when coadministered with gliclazide, in healthy but not diabetic rats. Eur J Drug Metab Pharmacokinet 2012;37(2):99-108.

7. Mikov M, Al-Salami H, Golocorbin-Kon S, Skrbic R, Raskovic A, Fawcett JP. The influence of 3alpha,7alpha-dihydroxy-12-keto-5beta-cholanate on gliclazide pharmacokinetics and glucose levels in a rat model of diabetes. Eur J Drug Metab Pharmacokinet 2008;33(3):137-42.
8. Mikov M, Đanić M, Pavlović N, Stanimirov B, Goločorbin-Kon S, Stankov K, et al. Potential applications of gliclazide in treating type 1 diabetes mellitus: formulation with bile acids and probiotics. Eur J Drug Metab Pharmacokinet 2018;43(3):269-80.

9. Davis TM, Daly F, Walsh JP, Ilett KF, Beilby JP, Dusci LJ, et al. Pharmacokinetics and pharmacodynamics of gliclazide in Caucasians and Australian Aborigines with type 2 diabetes. Br J Clin Pharmacol 2000;49:223-30.

10. McGavin JK, Perry CM, Goa KL. Gliclazide modified release. Drugs 2002;62(9):1357-64.

11. Al-Kassas RS, Al-Gohary OMN, Al-Faadhel MM. Controlling of systemic absorption of gliclazide through incorporation into alginate beads. Int $\mathrm{J}$ Pharm 2007;341(1-2):230-7.

12. Mooranian A, Negrulj R, Al-Sallami HS, Fang Z, Mikov M, Golocorbin-Kon S, et al. Release and swelling studies of an innovative antidiabetic-bile acid microencapsulated formulation, as a novel targeted therapy for diabetes treatment. J Microencapsul 2015;32(2):151-6.

13. Mooranian A, Negrulj R, Chen-Tan N, Al-Sallami HS, Fang Z, Mukkur T, et al. Novel artificial cell microencapsulation of a complex gliclazide-deoxycholic bile acid formulation: a characterization study. Drug Des Devel Ther. 2014;8:1003-12.

14. Mathavan S, Chen-Tan N, Arfuso F, Al-Salami H. A comprehensive study of novel microcapsules incorporating gliclazide and a permeation enhancing bile acid: hypoglycaemic effect in an animal model of type1 diabetes. Drug Deliv 2016;23(8):2869-80.

15. Mooranian A, Negrulj R, Mathavan S, Martinez J, Sciarretta J, Chen-Tan N, et al. Stability and release kinetics of an advanced gliclazide-cholic acid formulation: the use of artificial-cell microencapsulation in slow release targeted oral delivery of antidiabetics. J Pharm Innov 2014;9(2):150-7.

16. Mooranian A, Negrulj R, Mathavan S, Martinez J, Sciarretta J, Chen-Tan N, et al. An advanced microencapsulated system: a platform for optimized oral delivery of antidiabetic drug-bile acid formulations. Pharm Dev Technol 2015;20(6):702-9.

17. Golocorbin-Kon S, Calasan J, Milijasevic B, Vukmirovic S, Lalic-Popovic M, Mikov M, et al. High-loading dose of microencapsulated gliclazide formulation exerted a hypoglycaemic effect on type 1 diabetic rats and incorporation of a primary deconjugated bile acid, diminished the hypoglycaemic antidiabetic effect. Eur J Drug Metab Pharmacokinet 2017;42(6):1005-11.

18. Stepensky D, Friedman M, Raz I, Hoffman A. Pharmacokinetic-pharmacodynamic analysis of the glucose-lowering effect of metformin in diabetic rats reveals first-pass pharmacodynamic effect. Drug Metab Dispos 2002;30(8):861-8.

19. Marathe PH, Wen Y, Norton J, Greene DS, Barbhaiya $\mathrm{RH}$, Wilding IR. Effect of altered gastric emptying and gastrointestinal motility on metformin absorption. $\mathrm{Br}$ J Clin Pharmacol 2000;50(4):325-32.

20. Stětinová V, Květina J, Pastera J, Polásková A, Prazáková M. Gliclazide: pharmacokinetic-pharmacodynamic relationships in rats. Biopharm Drug Dispos 2007;28(5):241-8.

21. Resztak M, Hermann TW, Sawicki W, Danielak DZ. Pharmacokinetics and pharmacodynamics of gliclazide from immediate and modified release formulation tablets in rats. Iran J Pharm Res 2014;13(1):29-37. 